\title{
Efficient Hybrid Transform Scheme for Medical Image Compression
}

\author{
Aree Ali Mohammed \\ Computer Department \\ University of Sulaimani \\ Sulaimani, Iraq
}

\author{
Jamal Ali Hussein \\ Computer Department \\ University of Sulaimani \\ Sulaimani, Iraq
}

\begin{abstract}
In recent times, developing hybrid schemes for effective image compression has gained enormous popularity among researchers. This research paper presents a proposed scheme for medical image compression based on hybrid compression technique (DWT and DCT). The goal is to achieve higher compression rates by applying different compression thresholds for the wavelet coefficients of each DWT band (LL and HH) while DCT transform is applied on (HL and LH) bands with preserving the quality of reconstructed medical image. The retained coefficients are quantized by using adaptive quantization according to the type of transformation. Finally the entropy coding (variable shift coding) is used to encode the quantization indices. Experimental results show that the coding performance can be significantly improved by the hybrid DWTDCT algorithm.
\end{abstract}

\section{Keywords}

image compression; quasi lossless compression; adaptive quantization; hybrid scheme; DWT; DCT ;medical image.

\section{INTRODUCTION}

Wavelet transform provides numerous desirable properties, such as efficient multi-resolution representation, scalability, and embedded coding with progressive transmission, which are beneficial to the image compression applications [1]. Wavelet based multi-resolution representation matches the Human Visual System, specifically the higher detail information of an image is represented by the shorter basis function with higher spatial resolution and the lower detail information is represented by the larger basis function with higher spectral resolution [2]. The recently standardized image compression scheme known as JPEG 2000 uses discrete wavelet transform as the underlying transform algorithm [3]. Recently some hybrid coding techniques are developed. $\mathrm{H}$. Hsin et al proposed a hybrid algorithm using SPIHT and EBC (embedded block coding) to code low frequency and high frequency wavelet coefficients, respectively; the intermediate coding results of low frequency coefficients are used to facilitate the coding operation of high frequency coefficients [4]. Another hybrid scheme is proposed by combining Kohonen's Self Organizing Feature Map (SOFM) based Vector Quantization (VQ) coding and Set Partitioning in Hierarchical Trees (SPIHT) coding for effective compression of images [5].

In [6] a strategy to increase the compression ratio with simple computational burden and excellent decoded quality is presented. In [7] DCT and modified SPIHT algorithm are adopted to encode DCT coefficients. The proposed algorithm also provides the deblocking function in low bit rate in order to improve the perceptual quality. A lifting scheme wavelet-based transform with a modified entropy coding algorithm is proposed in [8-10]. It showed how block subband coding leads to increase the compression factor with preserve the quality.

In this paper, a novel algorithm for medical image compression is developed using 9/7 Tap wavelet filter, DCT transform and optimized entropy-based coding technique. The diagram of proposed work is shown in the Figure 1.

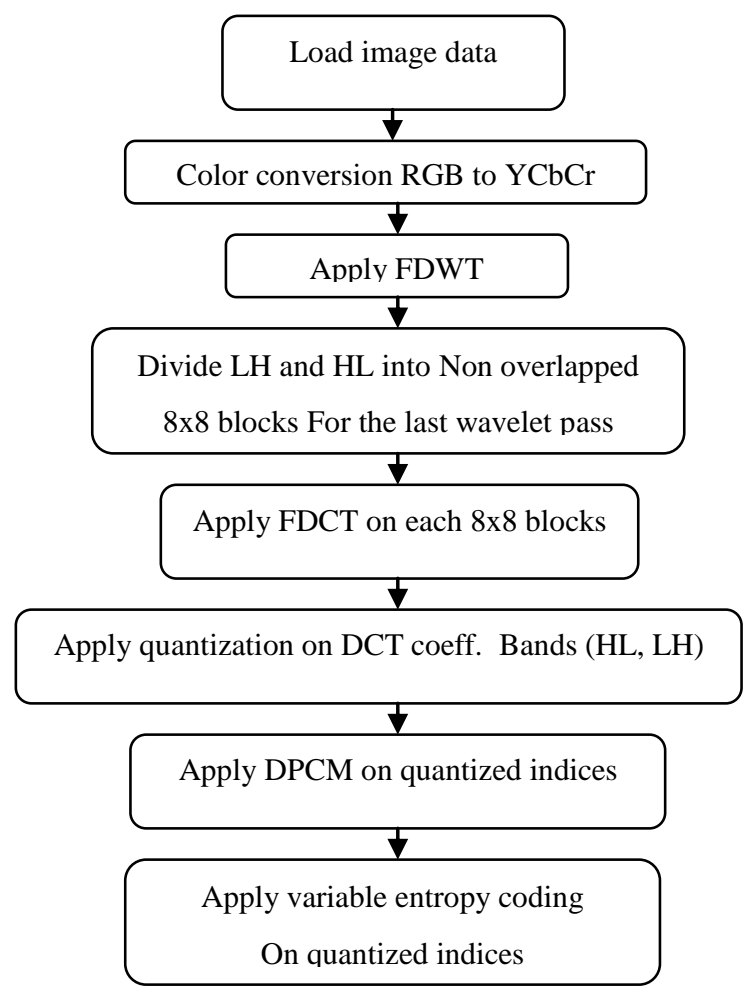

Fig 1: Proposed method diagram

In Sec-2, the proposed algorithm and its implementation are presented. In Sec-3, the conducted test which is evaluating the proposed scheme is detailed. Finally the main conclusions are summarized in Sec-4. 


\section{PROPOSED CODING ALGORITHMS STEPS}

In this section detail steps of the proposed medical image compression are proposed as follows:

\subsection{Color Space Conversion (RGB to $\mathrm{YCbCr})$ )}

$\mathrm{YCbCr}$ refers to the color resolution of digital component video signals, which is based on sampling rates. In order to compress bandwidth, $\mathrm{Cb}$ and $\mathrm{Cr}$ are sampled at a lower rate than $\mathrm{Y}$, which is technically known as "chroma subsampling." This means that some color information in the image is being discarded, but not brightness (luma) information.

$$
\begin{aligned}
& \mathrm{Y}=0.2989 * \mathrm{R}+0.5866 * \mathrm{G}+0.1145 * \mathrm{~B} \\
& \mathrm{Cb}=-0.1687 * \mathrm{R}-0.3312 * \mathrm{G}+0.5 * \mathrm{~B} \\
& \mathrm{Cr}=0.5 * \mathrm{R}-0.4183 * \mathrm{G}-0.0816 * \mathrm{~B}
\end{aligned}
$$

\subsection{Forward Discrete Wavelet Transform (FDWT)}

Discrete Wavelet transform (DWT) represents an image as a sum of wavelet functions (wavelets) with different locations and scales. Any decomposition of an image into wavelets involves a pair of waveforms: one to represent the high frequencies corresponding to the detailed parts of an image (wavelet function) and one for the low frequencies or smooth parts of an image (scaling function).

The filter which is used for this transformation is a non reversible filter (real to real transform 9/7Tap) and can only be used for lossy coding. In figure 2 the wavelet of 2 levels of decomposition is shown:

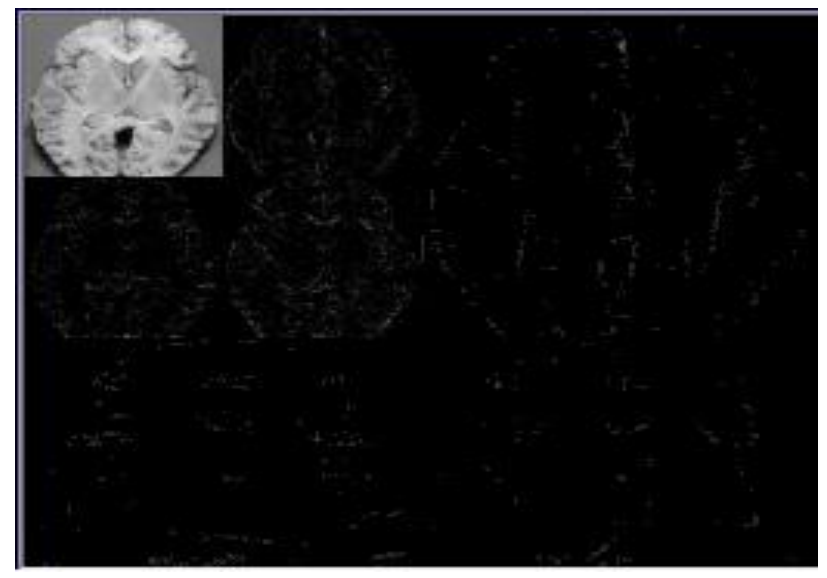

Fig 2: Wavelet decomposition (no. of pass = 2)

After applying FDWT on the medical image's data, one can obtain different level of bands. LL and HH band's coefficients are directly sent to the adaptive quantizer according to the nature of bands. The remaning bands (HL and LH) coefficients are subjected to DCT transformation.

\subsection{Forward Discrete Consine Transform (FDCT)}

Each of HL and LH bands are divided into 8X8 blocks and converted to frequency domain using 2D FDCT equation:

$$
\begin{aligned}
D(u, v)=\frac{1}{\sqrt{2 N}} & C(u) C(v) \\
& \sum_{x=0}^{N-1} \sum_{y=0}^{N-1} P(x, y) \cos \left[\frac{(2 x+1) u \pi}{2 N}\right] \cos \left[\frac{(2 y+1) v \pi}{2 N}\right]
\end{aligned}
$$

where

$$
C(i)=\left\{\begin{array}{lll}
1 / \sqrt{2} & \text { if } & \mathrm{i}=0 \\
1 & \text { if } & \mathrm{i}>0
\end{array}\right.
$$

\subsection{DCT Quantization}

DCT block's coefficients of Y component must be quantized using the following luminance quantization matrix:

The quantization is done by simply dividing each DCT's coefficient by its corresponding value in the quantization matrix and then rounding to the nearest integer.

$$
\left[\begin{array}{cccccccc}
16 & 11 & 10 & 16 & 24 & 40 & 51 & 61 \\
12 & 12 & 14 & 19 & 26 & 58 & 60 & 55 \\
14 & 13 & 16 & 24 & 40 & 57 & 60 & 59 \\
14 & 17 & 22 & 29 & 51 & 87 & 80 & 62 \\
18 & 22 & 37 & 56 & 68 & 109 & 103 & 77 \\
24 & 35 & 55 & 64 & 81 & 104 & 113 & 92 \\
49 & 64 & 78 & 87 & 103 & 121 & 120 & 101 \\
72 & 92 & 95 & 98 & 112 & 100 & 103 & 99
\end{array}\right]
$$

The $\mathrm{Cb}$ and $\mathrm{Cr}$ components are quantized using chrominance quantization matrix:

$$
\left[\begin{array}{llllllll}
17 & 18 & 24 & 47 & 99 & 99 & 99 & 99 \\
18 & 21 & 26 & 66 & 99 & 99 & 99 & 99 \\
24 & 26 & 55 & 99 & 99 & 99 & 99 & 99 \\
47 & 66 & 99 & 99 & 99 & 99 & 99 & 99 \\
99 & 99 & 99 & 99 & 99 & 99 & 99 & 99 \\
99 & 99 & 99 & 99 & 99 & 99 & 99 & 99 \\
99 & 99 & 99 & 99 & 99 & 99 & 99 & 99 \\
99 & 99 & 99 & 99 & 99 & 99 & 99 & 99
\end{array}\right]
$$

In the resulting matrix many of the higher frequency components are rounded to zero, and many of the rest become small positive or negative numbers.

\subsection{DWT Quantization}

The LL, HH coefficients must be quantized using adaptive quantization. The luminance component $\mathrm{Y}$ requires the small step of quantization while $\mathrm{Cb}$ and $\mathrm{Cr}$ need a large step. After this step, a large sequence of zeros is obtained especially in $\mathrm{HH}$ part of the image. 


\subsection{DPCM and Mapping to Positive}

Basic concept of DPCM - coding a difference, is based on the fact that most source signals show significant correlation between successive samples so encoding uses redundancy in sample values which implies lower bit rate.

The forward differential pulse code modulation is applied on the quantized (LL band) wavelet coefficients and quantized DC coefficients of DCT transform. And then all the coefficients must be converted into positive values by mapping to positive technique. The block diagram of these steps is shown as in figure 3.

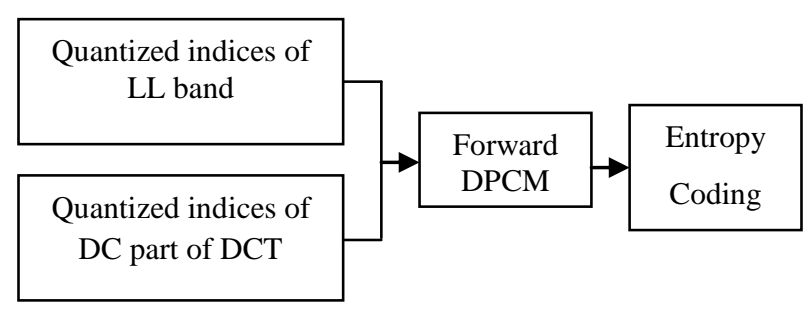

Fig 3: Block diagram of DPCM and Mapping

\subsection{Variable Entropy Coding}

The proposed coding scheme is a variable shift coding technique which gives a few bits to the short codeword and many bits to the long codeword. The main idea behind the shift coding algorithm is to find the maximum hybrid transform coefficients in the data set and optimized these coefficients to take a small numbers of bits. The other coefficients within the set are coded with the same number of bits [8].

\section{DECODING ALGORITHM}

The reconstructed image is obtained by applying the inverse steps of coding process. Figure 4 and 5 show the coding and decoding processes' steps.

\section{TEST RESULTS}

The tests are performed on medical images taking by MRI with two different sizes, brain (256X256) and pulmonary (512x512) see figure 6 . To show the effect of involved parameters on the compression ratio, different values of scaling factor ( $\alpha$ ) are used. $\alpha$ affects the quantization steps (QY and $\mathrm{QCb}, \mathrm{QCr}$ ) for both DWT and DCT coefficients, see figure 7. Table 1 and 2 present the test results for the number of pass 2 and 3 respectively. The quatization parameters are fixed as $\mathrm{QY}=35$, $\mathrm{QCb}=40, \mathrm{QCr}=40$.

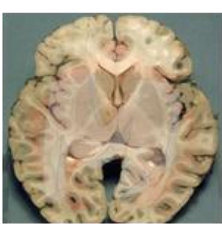

Brain

$(265 \times 256)$

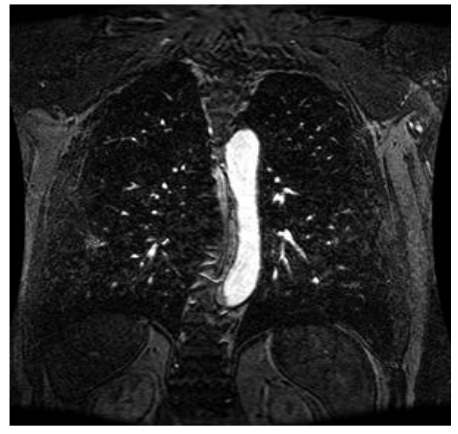

Lung

$(512 \times 512)$
Fig 6: Image used in tests

\begin{tabular}{|c|c|c|c|c|}
\hline \multicolumn{5}{|c|}{ Table 1. Resulting parameters where no. of pass = 2 } \\
\hline \multirow{4}{*}{ Image } & $\begin{array}{c}\text { DWT } \\
\text { Quantization } \\
\text { factor }\end{array}$ & $\begin{array}{c}\text { DCT } \\
\text { Quantization } \\
\text { factor }\end{array}$ & $\begin{array}{c}\text { Compression } \\
\text { ratio }\end{array}$ & PSNR \\
\hline \multirow{4}{*}{ brain } & 0.2 & 0.2 & 28.88 & 35.96 \\
\cline { 2 - 5 } & 0.5 & 0.5 & 42.78 & 34.24 \\
\cline { 2 - 5 } & 1.0 & 1.0 & 58.15 & 32.41 \\
\hline \multirow{3}{*}{ lung } & 0.2 & 0.2 & 28.10 & 35.79 \\
\cline { 2 - 5 } & 0.5 & 0.5 & 42.59 & 33.73 \\
\cline { 2 - 5 } & 1.0 & 1.0 & 59.66 & 31.25 \\
\hline
\end{tabular}

Table 2. Resulting parameters where no. of pass $=3$

\begin{tabular}{|c|c|c|c|c|}
\hline \multirow{4}{*}{ Image } & $\begin{array}{c}\text { DWT } \\
\text { Quantization } \\
\text { factor }\end{array}$ & $\begin{array}{c}\text { DCT } \\
\text { Quantization } \\
\text { factor }\end{array}$ & $\begin{array}{c}\text { Compression } \\
\text { ratio }\end{array}$ & PSNR \\
\cline { 2 - 5 } & 0.2 & 0.2 & 17.7 & 35.8 \\
\cline { 2 - 5 } & 0.5 & 0.5 & 32.9 & 33.2 \\
\hline \multirow{3}{*}{ lung } & 1.0 & 1.0 & 51.9 & 29.2 \\
\cline { 2 - 5 } & 0.2 & 0.2 & 21.89 & 35.7 \\
\cline { 2 - 5 } & 1.0 & 0.5 & 34.89 & 33.1 \\
\hline
\end{tabular}




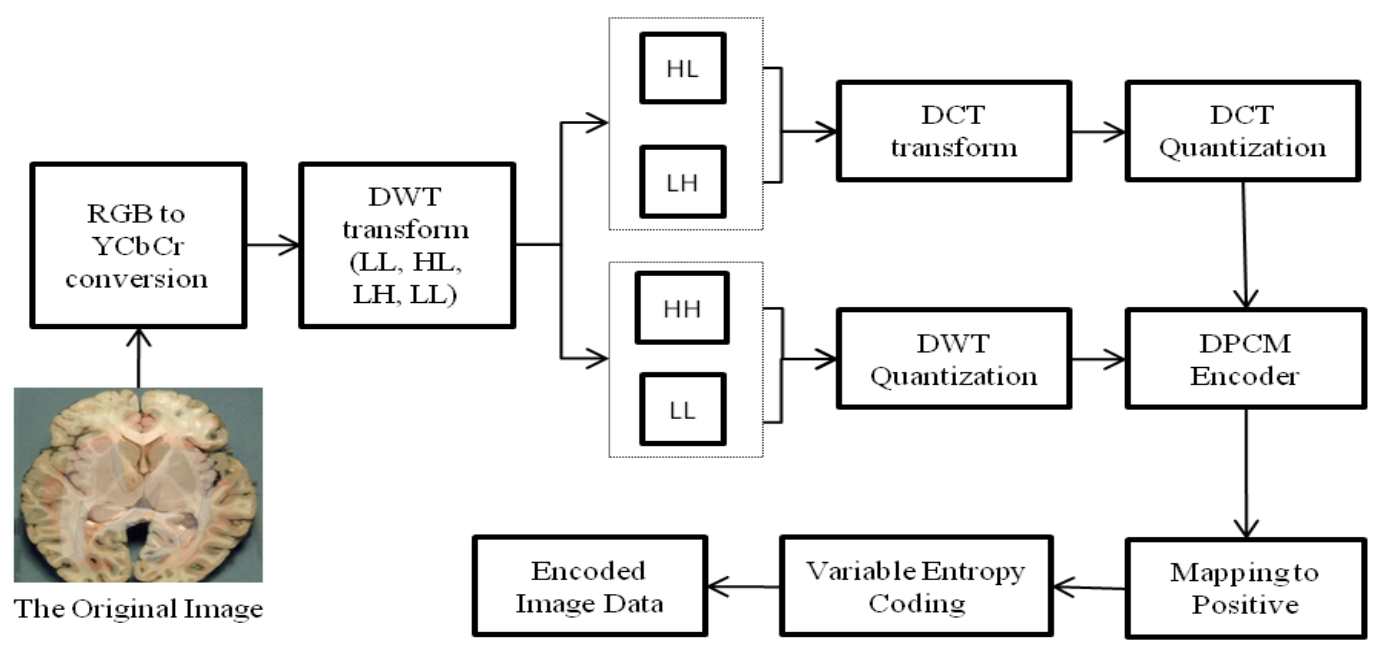

Fig 4: Coding Process

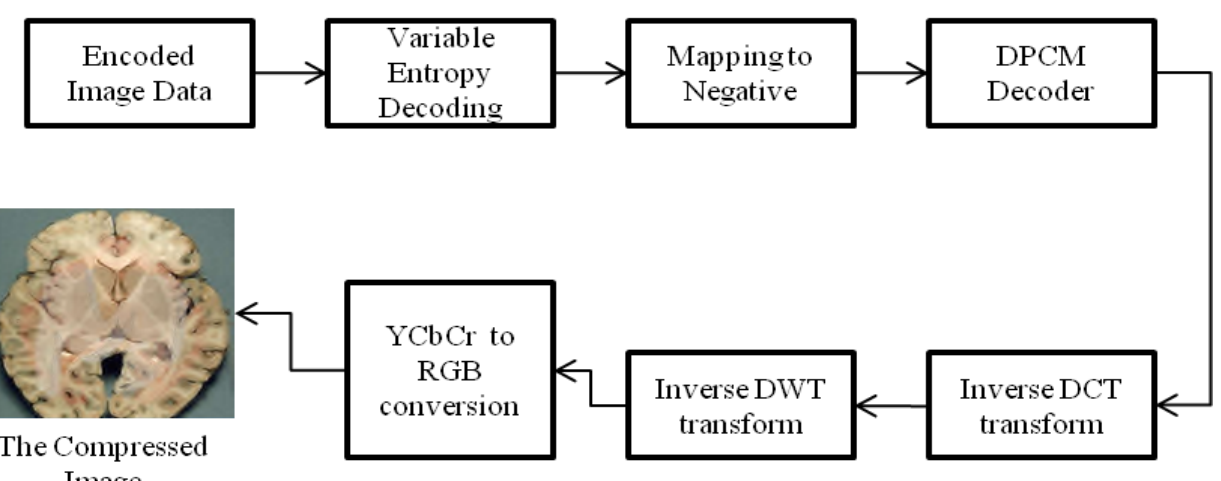

Fig 5: Decoding Process
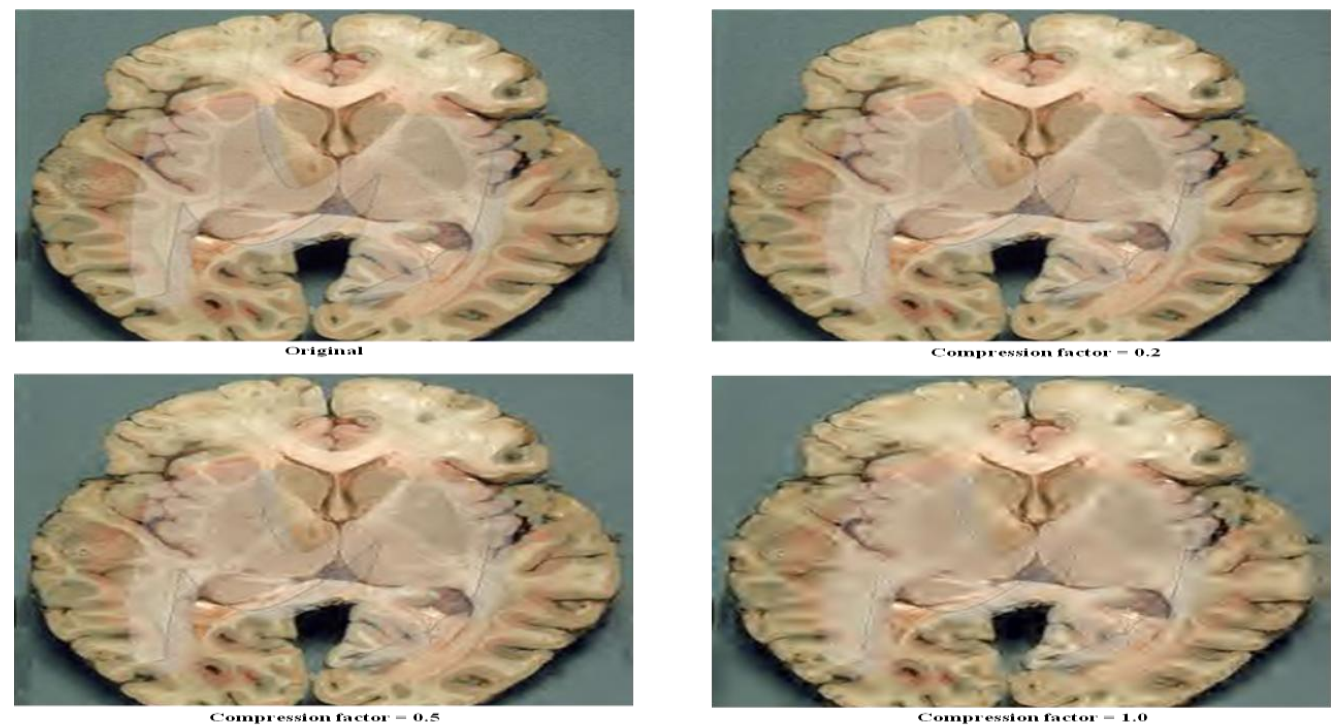

Fig 7: Compressed images with different compression factors and number of DWT passes = 2 


\section{CONCLUSIONS}

In this paper a new hybrid scheme for medical image compression is proposed. This method is based on both DWT and DCT compression techniques. This hybrid compression technique is tested against different medical images using different values of compression factors (i.e. DWT and DCT quantization factors). As the quantization factors increase the Compression ratio increase and the quality measurement (PSNR) decrease.

Experimental results show that these compressed medical images preserve its quality where quantization factor is less than 0.5 . Where quantization factor is larger than 0.5 , the constructed image after compression will begun slowly losing its quality.

\section{REFERENCES}

[1] M. Antonini, M. Barlaud, P. Mathieu, and I. Daubechies, "Image Coding Using Wavelet Transform", ieee trans. on image processing, vol. 1, april 1992, pp. 205-220.

[2] G. Strang and T. Nguyen, "Wavelets And Filter Banks", Wellesley-Cambridge Press (1996).

[3] “JPEG 2000 Image Coding System”, ISO/IEC CD15444-1: 1999 (Version 1.0).

[4] Hsi-Chin Hsin, Jenn-Jier Lien, and Tze-Yun Sung, "A Hybrid SPIHT-EBC Image Coder", IAENG International Journal of Computer Science, Volume 34, Issue 1, 2007.

[5] D. Rawat, S. Meher, "A Hybrid Coding Scheme Combining SPIHT and SOFM Based Vector Quantization for Effectual Image Compression", European Journal of Scientific Research Vol.38 No.3 (2009), pp.425-440.

[6] Yung-Gi Wu and Shen-Chuan Tai, "Medical image compression by discrete cosine transform spectral similarity strategy". IEEE Transactions on Information Technology in Biomedicine, $2001 \mathrm{Sep}$, volume 5, issue 3, pp. 236-243.

[7] Wen-Chien Yan and Yen-Yu Chen, "DCT-Based Image Compression Using Wavelet-Based Algorithmwith Efficient Deblocking Filter", 14th European Signal Processing Conference (EUSIPCO 2006), Florence, Italy, September 4-8, 2006.

[8] Loay A. George, Aree A. Mhammad, "Intra Frame Compression Using Lifting Scheme Wavelet-Based Transformation (9/7-Tap Cdf Filter)", International Conference on Multimedia Systems and Applications, MSA 2007, June 25-28, 2007, Las Vegas Nevada, USA.

[9] Singh S., Kumar V and Verma HK.," DWT-DCT hybrid scheme for medical image compression.”, Journal of Medical Engineering \& Technology, Vol.31 No. 2, 2007, pp. 109-122.

[10] Suchitra S. and Khan A., "A Sub-sample Based Hybrid DWT-DCTAlgorithm for Medical Imaging Applications", Journal of Selected Areas in Bioengineering, 2010. 